\title{
Review: clinical findings should determine choice of imaging test for patients with low back pain in a primary care setting
}

Jarvik JG, Deyo RA. Diagnostic evaluation of low back pain with emphasis on imaging. Ann Intern Med 2002;137:586-97.

Sources of funding: Agency for Healthcare Research and Quality and National Institute of Arthritis and Skin Diseases.

For correspondence: DrJ G Jarvik, University of Washington, Seattle WA, USA. jarvikj@ u.washington.edu

\section{QUESTION: In patients with low back pain in a primary care setting, what is the diagnostic accuracy of clinical information and imaging?} Musculoskeletal and

\section{Data sources}

Studies were identified by searching Medline (January 1966 to September 2001).

\section{Study selection}

Studies were selected if they described the accuracy of plain radiography, computed tomography (CT), magnetic resonance imaging (MRI), or bone scanning in

Estimated diagnostic accuracy of imaging techniques for lumbar spine conditions*

\begin{tabular}{|c|c|c|c|c|}
\hline \multirow{2}{*}{$\begin{array}{l}\text { Imaging } \\
\text { techniques }\end{array}$} & \multicolumn{2}{|l|}{ Herniated disc } & \multicolumn{2}{|l|}{ Cancer } \\
\hline & Sensitivity & Specificity & Sensitivity & Specificity \\
\hline $\begin{array}{l}\text { Computed } \\
\text { tomography }\end{array}$ & $62 \%$ to $90 \%$ & $70 \%$ to $87 \%$ & Data unavaila & ble \\
\hline $\begin{array}{l}\text { Magnetic resonance } \\
\text { imaging }\end{array}$ & $60 \%$ to $100 \%$ & $43 \%$ to $97 \%$ & $83 \%$ to $93 \%$ & $90 \%$ to $97 \%$ \\
\hline Plain radiography & Data unavailable & & $60 \%$ & $95 \%$ to $96 \%$ \\
\hline
\end{tabular}

*Estimated based on multiple studies included in the review.

\section{COMMENTARY}

The review by Jarvik $e t$ al is a useful guide for primary care professionals who are responsible for the management of patients with low back pain. Although the emphasis is on the accuracy of imaging, the authors include a table on the accuracy of the history and an algorithm for the diagnostic evaluation of patients with low back pain. The latter table includes the appropriate criteria and timing for imaging.

A concern for practitioners is missing a diagnosis that requires urgent intervention or missing an underlying systemic disease or neurological impairment that requires surgical evaluation. The pretest probability for all of these is low. The authors noted that, using guidelines such as those of the Agency for Health Care Policy and Research, imaging may not be required for acute back pain of $<6$ weeks duration unless there is a high suspicion for systemic disease or progressive neurological deficit. ${ }^{1}$

The review did not attempt a meta-analysis because of concerns about the heterogeneity of the results. They were also concerned that the biases in the original studies may have impaired estimates of accuracy.

Although plain radiography is the most common spinal imaging technique, it was less sensitive for cancer, infection, and ankylosing spondylitis than CT, MRI, or radionuclide scanning. In the review, plain radiography was only superior in specificity for cancer when compared with the other techniques. For systemic disease, MRI showed the best sensitivity and specificity, and MRI and CT had about the same specificity and sensitivity for degenerative lesions with neurological changes.

Although not addressed by the authors, the findings suggest that little indication for plain radiography in the management of low back pain exists. These results would have to be confirmed by other studies addressing the methodological problems outlined by the authors; however, the importance of reducing the requirements for imaging is well recognized.

Edward S Gibson, MD Hamilton Hospitals Assessment Centre Hamilton, Ontario, Canada

1 Bigos S, Bowyer O, Braen G, et al. Acute low back problems in adults. Clinical Practice Bigos S, Bowyer O, Braen G, et al. Acute low back problems in adults. Clinical Practice
Guideline No 14. AHCPR Publication No. 95-06742. Rockville, MD: Agency for Health Guideline No 14. AHCPR Publication No. patients with low back pain. Paediatric studies and studies of myelography, discography, and positron emission tomography were excluded.

\section{Data extraction}

Extracted data included diagnostic results and methodological quality.

\section{Main results}

73 studies were included. The estimated diagnostic accuracy of plain radiography, CT, and MRI for herniated disc and cancer are displayed in the table. For vertebral metastatic lesions, plain radiography was less sensitive than other imaging tests. In primary care patients, the presence of a lytic or blastic lesion on plain radiographs had a sensitivity of $60 \%$ and a specificity of $99.5 \%$ for cancer. For vertebral infection, radiography had low specificity; in 1 study, radiography had a sensitivity of $82 \%$ and a specificity of $57 \%$ for osteomyelitis. Sacroiliitis occurs early in ankylosing spondylitis and can be detected by radiography. In 1 study, radiography had $45 \%$ sensitivity and $100 \%$ specificity for spondyloarthropathy. CT is a key spinal imaging test. In a meta analysis, CT showed sensitivities ranging from $70-100 \%$, and specificities ranging from $80-96 \%$ for the diagnosis of stenosis. In 1 study, MRI had a sensitivity of $96 \%$ and a specificity of $92 \%$ for detecting spinal infections. MRI had 81-97\% sensitivity and 72-100\% specificity for detecting stenosis. Bone scanning has been used to detect occult fractures, infections, or bony metastases. Studies using planar imaging to detect metastases reported estimated sensitivities between $74 \%$ and $98 \%$ and specificities between $64 \%$ and $81 \%$. For detecting infection, bone scanning was sensitive (90\%) but modestly specific (78\%) in 1 study. In 1 study, bone scanning had a sensitivity of $26 \%$ and a specificity of $100 \%$ for spondyloarthropathy.

\section{Conclusions}

In patients with low back pain in a primary care setting, estimates of the diagnostic accuracy of common imaging tests (plain radiography, computed tomography $[\mathrm{CT}]$, magnetic resonance imaging $[\mathrm{MRI}]$, and bone scanning) are limited by study biases. For patients with systemic diseases, MRI may have the greatest sensitivity and specificity. For patients with degenerative conditions causing neurological impairment, MRI and CT may have similar accuracy. In the absence of systemic disease or progressive neurological deficits, imaging is usually not required in patients with acute back pain of $<6$ weeks' duration. 